\title{
Growth-Driven Percolations: The Dynamics of Community Formation in Neuronal Systems
}

\author{
Luciano da Fontoura Costa \\ Institute of Physics of São Carlos. University of São Paulo, \\ São Carlos, SP, PO Box 369, 13560-970, phone +55 163373 9858, \\ FAX +551633713616, Brazil, luciano@if.sc.usp.br \\ Regina Célia Coelho \\ Faculdade de Ciências Matemáticas, da Natureza e Tecnologia de Informação - Methodist University of Piracicaba, \\ Rodovia do Açúcar, km 156 - Campus Taquaral - Taquaral, Piracicaba, SP, \\ Brazil, 13400 - 901 phone +55193124 1515 - Ext. 1245, Fax +55193124 1701
}

(Dated: October 29, 2018)

\begin{abstract}
The quintessential property of neuronal systems is their intensive patterns of selective synaptic connections. The current work describes a physics-based approach to neuronal shape modeling and synthesis and its consideration for the simulation of neuronal development and the formation of neuronal communities. Starting from images of real neurons, geometrical measurements are obtained and used to construct probabilistic models which can be subsequently sampled in order to produce morphologically realistic neuronal cells. Such cells are progressively grown while monitoring their connections along time, which are analysed in terms of percolation concepts. However, unlike traditional percolation, the critical point is verified along the growth stages, not the density of cells, which remains constant throughout the neuronal growth dynamics. It is shown, through simulations, that growing beta cells tend to reach percolation sooner than the alpha counterparts with the same diameter. Also, the percolation becomes more abrupt for higher densities of cells, being markedly sharper for the beta cells.

PACS numbers: 89.75.Fb, 87.18.Sn, 02.10.Ox, 89.75.Da, 89.75.Hc, 2.50.Ey, 2.50.Ng
\end{abstract}

The brain is a world consisting of a number of unexplored continents and great stretches of unknown territory. (Santiago Ramon$y$-Cajal)

Neurons can be understood as cells which, along the evolutionary process, have become highly specialized for establishing connections between themselves along a wide range of spatial scales (ranging from microns to meters). In order to minimize metabolism and allow connections to selective targets, neurons acquired their intricated, ramified shapes. Indeed, instead of implementing casual connections with every surrounding cell, a neuron links to specific targets which can be nearby in the same neuronal region or far away in another cortical area originating, in the process, the basic architecture required for proper operation of the central nervous system. Interestingly, the connectivity pattern of a mature neuronal system is determined not only by the genome, which is unable to code all connections [21], but predominantly by the history of neuronal activity under stimuli presentation. Neurons are produced at ventricular zones of the neuroepithelium, in the form of neuroblasts, which therefore differentiate and migrate to specific target regions and start to unfold their dendritic and axonal processes 1]. As such structures develop and extend towards specific targets, which occurs under the guidance of trophic factors, they synapse and start forming communities (or clusters) of connected cells, organized in specific ways so as to achieve proper operation. Indeed, the functional properties of such structures are to a large extent related to the underlying connecting patterns, implying that one of the fundamental problems in neuroscience is to understand how neuronal connections are established during the development of the central nervous system [1] .

Since connectivity is the main purpose underlying neuronal growth and organization, it is interesting to obtain suitable mathematical structures and relationships capable of representing and modeling the development of neuronal systems at a high level of morphological realism. While graphs/networks, where neurons are assigned to nodes and synapses to edges, provide a natural means to express the neuronal connections, several concepts from statistical mechanics can be used to model and simulate the connection dynamics. By providing an interesting interface between graph theory and statistical mechanics, the recent area of complex networks [2, 3, 4] represents a particularly promising perspective to bridge the gap between the morphology and dynamics of neuronal systems. In particular, the concept of percolation [5] stand out as particularly relevant for such investigations. Previous related works include the statistical physics investigation of scaling properties and the degree of separation in cortical networks [6], the small-world characterization of neuronal structures grown in vitro [7], the use of critical percolation point for neuronal shape characterization [8], and the identification of electrically active clusters in neural networks [9]. Related works addressing the relationship between neuronal geometry and function can also be found in the literature (e.g. [10], 11], 12], 13]). While such works have considered stactic neuronal shapes, the 


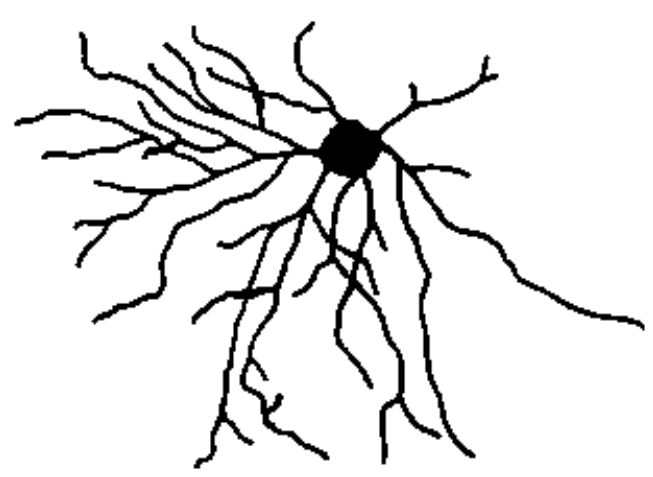

(a)

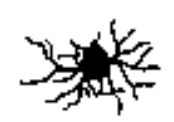

(b)

FIG. 1: Example of alpha (a), 15], and beta (b) 16] cells used in this work. Reproduced with permission.

development of a framework to model neuromorphically realistic neurons reported in [14] allows investigations of the neuronal connectivity during simulated neuronal development, by monitoring the size and other properties of the existing clusters in terms of time. Such a perspective motivated the extension of the concept of percolation to consider growing structures where the shapes of the objects may vary with time, a possibility proposed and investigated possibly for the first time in the present work.

This article starts by describing how the neuronal cells are represented and statistically modeled in terms of probabilities and follows by presenting the simulation of neuronal growth by using the Monte Carlo approach, as well as the characterization of the obtained structures in terms of the maximum cluster size observed along time. Such issues are illustrated with respect to a database of 2D neuronal cells including cat retina ganglionar cells of the types alpha (23 samples) and beta (27 samples), of which typical cells are illustrated in Figure 1

\section{NEURONAL MODELING}

One first key isse in neuromorphic modeling regards how to represent mathematically the geometry of neurons. While the typically observed diversity of shapes for the same class of cells immediately implies the use of statistics, the choice of the best (in the sense of being the most compact) set of morphometric parameters capable of representing the neuronal shape without considerable loss of information remains a challenging issue [13. The methodology for $2 \mathrm{D}$ neuronal representation adopted in this work follows the framework reported in

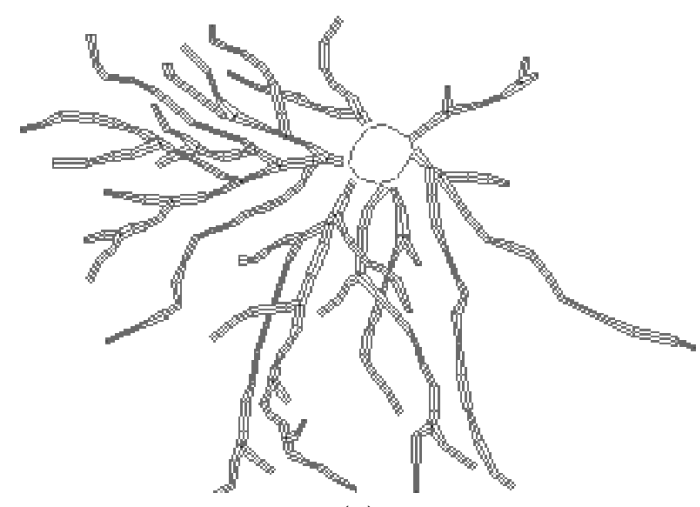

(a)

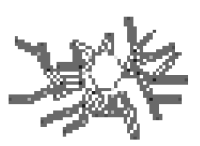

(b)

FIG. 2: Traced cells obtained from the cells in Figure $\square$ by using a customized neural tracer.

14], involving a probabilistic model considering the number of branches, the angles between them, the length of the dendritic and axonal segments, branching probability, and the length and angle of arcs of each branch. Therefore, the first step is to obtain such measurements from images of the real neuronal cells to be modeled. Typically, the cells are histologically marked and prepared, mounted on slides, and the respective images acquired through a camera interfaced to a light transmission (or fluorescence) microscope. The neurons in such images are then identified and isolated (e.g. 17]), producing binary representations (i.e. images containing only the neuronal cell - marked as one, and the background - marked as zero). An alternative way to obtain the binary images of the neural cells is through camera-lucida drawings, as is the case for the images in Figure 1 Once such binary images are obtained, their boundaries are extracted by using a customized neural tracer 22, producing results such as those illustrated in Figure 2 which corresponds to the boundaries of the cells in Figure 1

The dendrites are henceforth understood as trees, so that the respective hierarchical level can be precisely defined while considering the soma as reference. Therefore, the dendritic segments directly connected to the soma, as well as branching points initiating at such segments, are identified as being at hierarchical level 1 , and so on. Our simulations are restricted to a maximum of 10 hierarchical levels, as there are very few branchings occurring at higher levels in the real cells. The probability of branch points for each considered type of cell are shown in Figure 3(a) and (b), respectively for alpha and beta cells. The probability of the number of dendritic segments directly attached to the soma (i.e. hierarchical level 1) is also necessary for the statistical model of the growing 

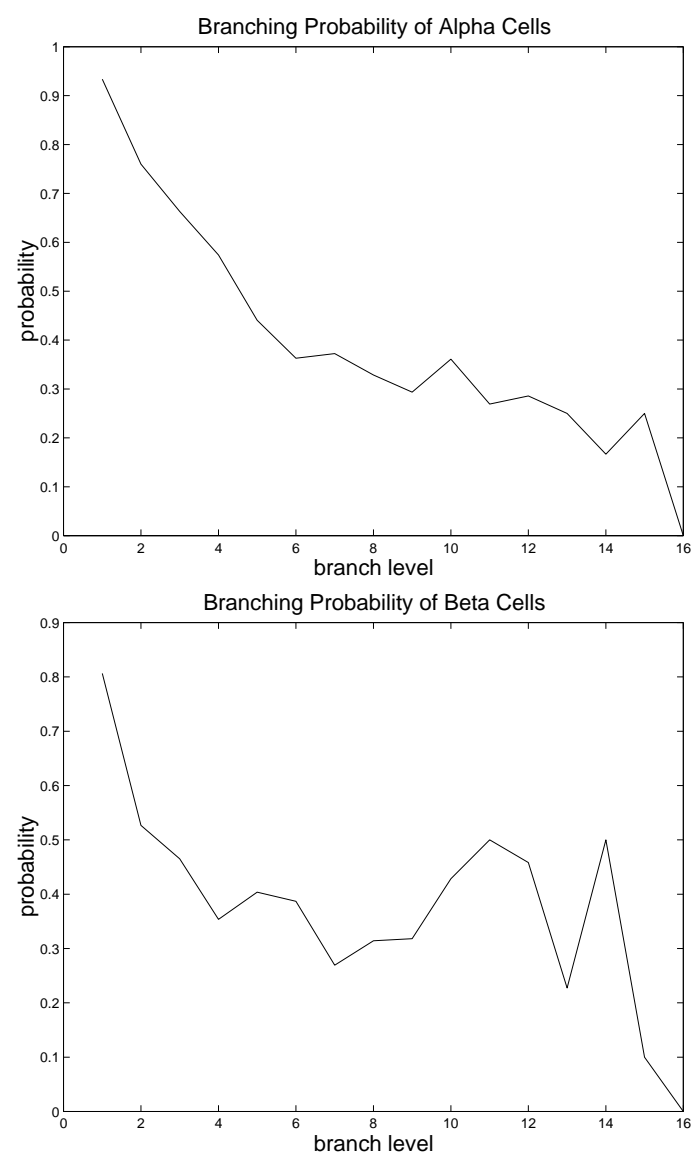

FIG. 3: Probability function illustrating the branching probability of alpha (a) and beta (b) cells.

cells. Figure 4 show the cumulative densities for the alpha (a) and beta (b) neuronal types.

In addition to being essential for neuronal shape modeling, the above branching and initial densities provide interesting information by themselves. For instance, it is clear from the two densities in Figure 3 that the alpha cells are characterized by higher branching rates at the lower hierarchical levels, as is clear from the more accentuated decreasal of the respective density along the hierarchical levels. Figure 4 presents the probability of the number of primary dendritic segments for alpha (a) and beta (b) cells. These graphs indicate that the beta cells are more likely to branch than alpha cells at hierarchical levels between 8 and 12 .

In addition to the above probabilities, it is also necessary to obtain probabilistic models of the dendritic segment arc-lengths. Although alpha cells are typically much larger than beta cells (especially in the periphery of the retina), we used size-normalized versions of the considered neurons in order to have neurons with similar sizes. This allows our percolation study to be mostly defined by the shape intrincacy of the cells rather than their sizes [23]. Figure 5] shows the cumulative twovariated density of such lengths in terms of the hierar-
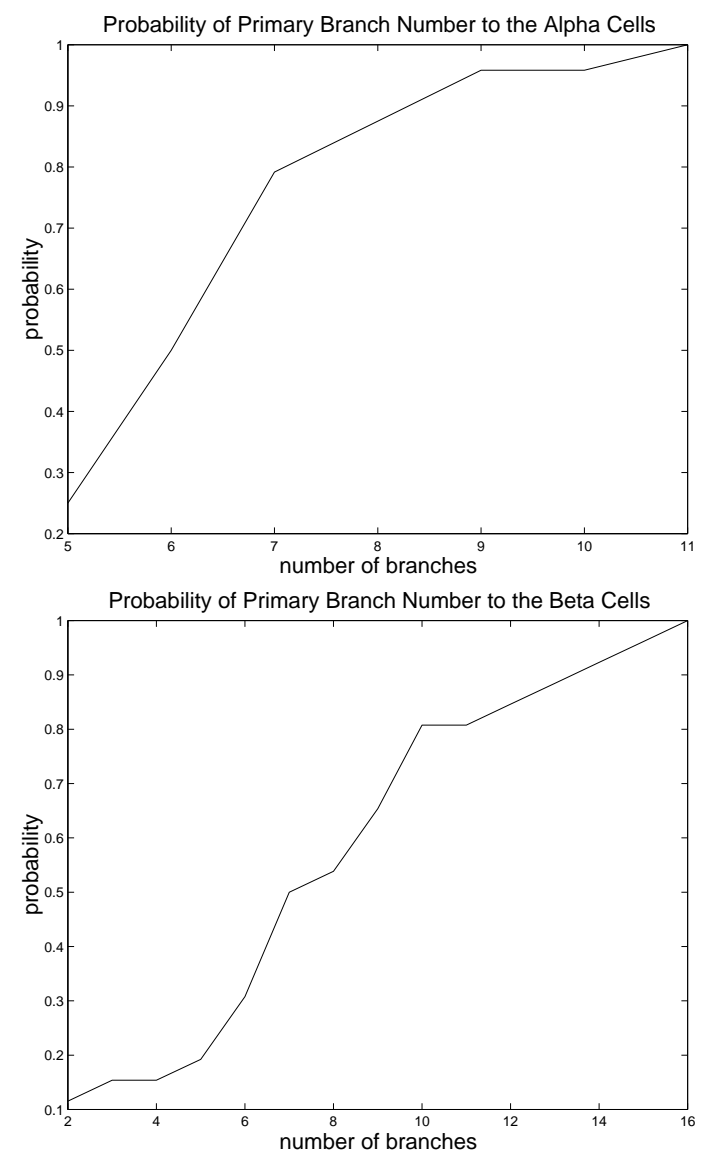

FIG. 4: Probability function of the number of primary dendritic segments to the alpha (a) and beta (b) cells.

chical level for the alpha (a) and beta (b) types of cells, while Figure 6 presents the angles of these dendritic segment arc-lengths for the alpha (a) and beta(b) cells. The last features considered in this work refer to the branch lengths and angles at the branch points, which are shown in Figures 7 and 8 for the alpha (a) and beta (b) cells. By "branch length" it is understood the total arc-length while moving from the branching point to the cell soma.

Note that both branching angle densities (i.e. Figures 6 and (7) are similar for both alpha and beta cells. The length-related densities (i.e. Figures 5 and 8) were obtained for alpha cells and then normalized with respect to their respective diameters (i.e. the largest distance between any two points of each cell) in such a way that they have the same average diameter as beta cells. Such a normalization was adopted so that the percolation only reflects the shape (and not size) of the cells.

\section{NEURONAL SYNTHESIS}

In order to generate the neuronal shapes, the probabilistic model of the neuronal geometry described in the previous section was statistically sampled by the Monte 

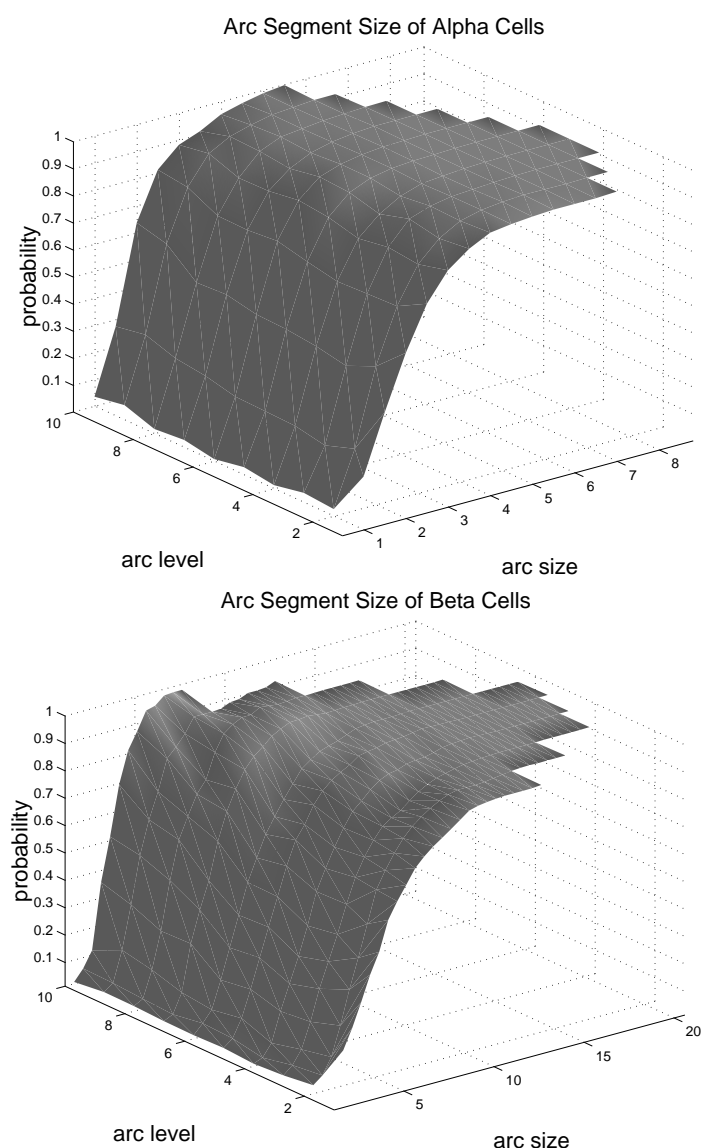

FIG. 5: Distribution function of dendritic segments arclengths to the alpha (a) and beta (b) cells.

Carlo approach as explained in the following. Initially, the soma of each cell was uniformly (Poisson distribution) distributed along an $N \times N$ matrix (associated to a digital image). The number of branches emerging from the soma was randomly chosen according to the respective density, being uniformly distributed along the somata, which are circular. For each cell, for each branch, the orientation of the emerging segment was drawn from the respective distribution. Straight segments are then incorporated, piece-by-piece, into the growing process. The length and orientation of each of these segment pieces was sampled through Monte Carlo from the respective statistical model, therefore taking into account the previous angle and length. In order to allow all neuronal cells to grow in a 'simultaneous' fashion, a single segment piece is incorporated into each growing branch, for each neuronal cell, at a time ('round-robin' scheme). Every time a new branch was visited, the probability for new branch or growth termination was sampled, and the respective action taken. In case we have a branch 24], the orientations of the two branching new segments were sampled from the respective distributions, and those branches were subsequently included in the 'round-robin' growth scheme. The growth of branches continued until one of the fol-
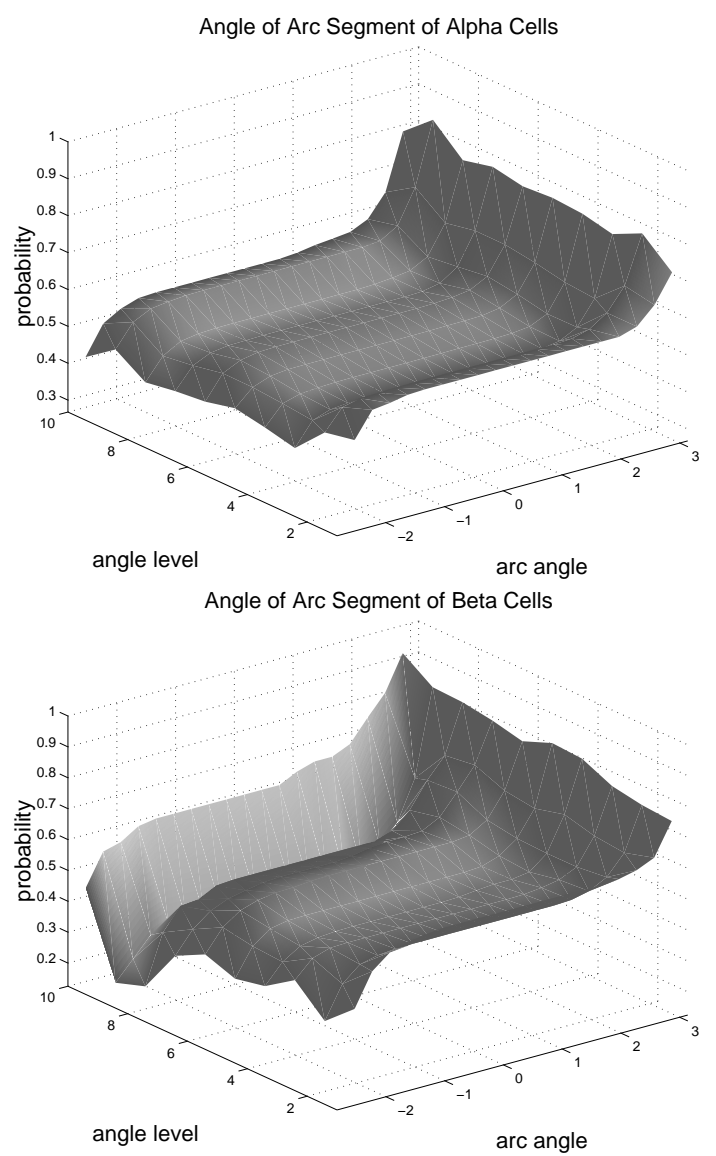

FIG. 6: Distribution function of dendritic segments arc-angles to the alpha (a) and beta (b) cells.

lowing conditions is reached: (a) it is selected for interruption; or (b) it reached 10 hierarchical stages. Figure 9 illustrates morphologically-realistic neuronal networks obtained by the growing process described above considering alpha (a) and beta (b) cells.

\section{PERCOLATION DYNAMICS}

During the simulated neuronal growth, a synaptic connection is implemented every time a growing dendrite overlaps any portion of the other current cells. So, as the cells develop in size and shape, they tend to form more connections. A group of connected cells is henceforth understood as a cluster. A natural representation of such growing structures can be immediately obtained by using graphs whose nodes correspond to the neuronal cell soma and the edges correspond to the synaptic connections. While several topological and morphometrical properties of the evolving neuronal networks can be quantified, in this work attention is concentrated on the size $S(t)$ of the cluster containing the maximum number of nodes i.e. the dominating cluster - found at each time instant $t$ (i.e. the growing stage). The sizes $S(t)$ are calculated 


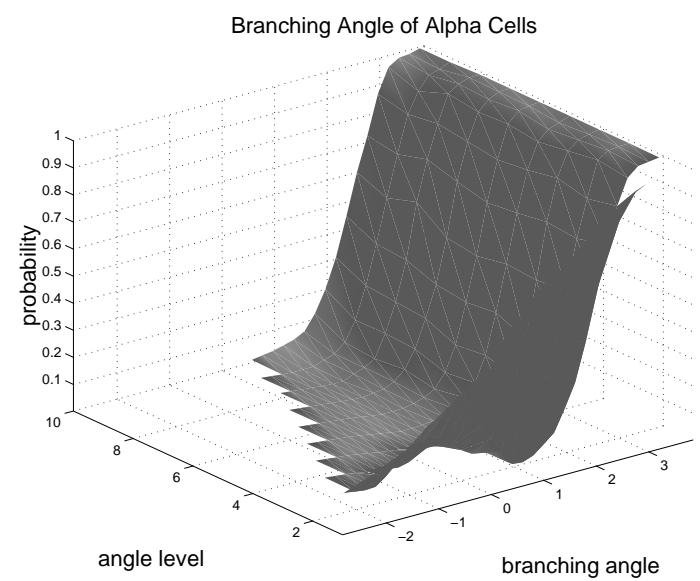

Branching Angle of Beta Cells

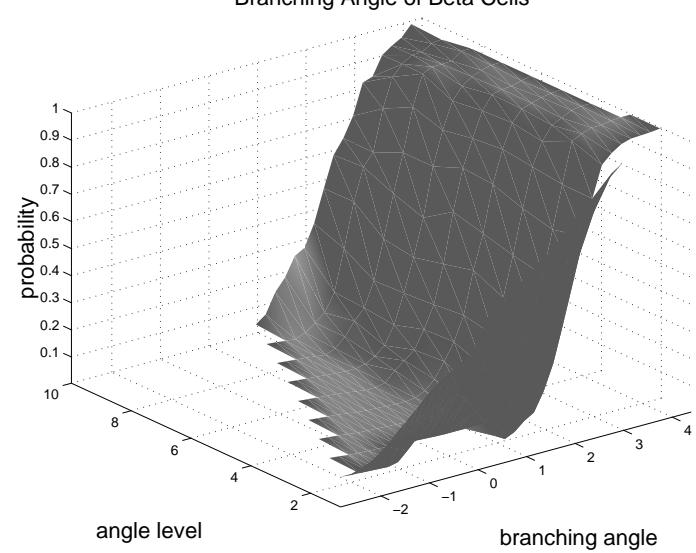

FIG. 7: Distribution function of the angles at the branch point to the alpha (a) and beta (b) cells.

from the graphs which are constructed as the networks evolve. The critical phenomenon of percolation is identified by looking for an abrupt transition along $S(t)$, which is related to the formation of the giant cluster [5]. After this point, the growing neuronal structure is characterized by the presence of such a giant community, which dominates the subsequent connectivity dynamics.

A single model of cell was considered in each simulation in order to keep statistical variability low and allow a more precise identification of the percolation critical point (not a density as in traditional percolation theory, but a time instant during the neuronal outgrowth). The chosen neuronal model is "stamped" $N$ times on the considered space (a rectangular window of 1000 by 1000 elements) according to the uniform probability. A total of 500 realizations was performed for each considered configuration, from which the average and standard deviation shown in the graphs were obtained. In order to avoid intense superposition between cells, cells were placed at least 5 pixels apart one another.

Figure 10(a-d) presents the evolution of the maximum cluster size considering growing densities of alpha cells, while Figure 10 (e-h) presents analogous graphs considering beta cells. As expected, the critical transition tends
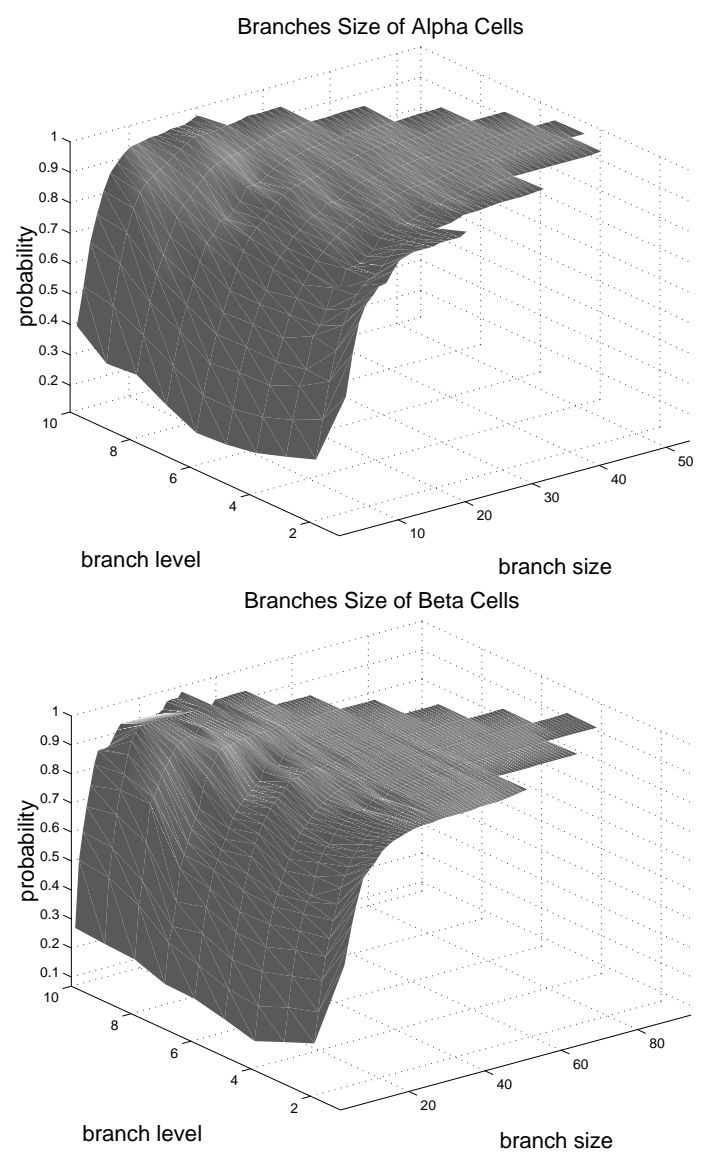

FIG. 8: Distribution function of the branch lengths to the alpha (a) and beta (b) cells.

to increase with the density of neurons, with markedly sharper transition being verified for the beta neuronal cells. More interestingly, it is also clear from the obtained results that the alpha cells implied longer times (e.g. higher cell densities) before percolation. Generally, percolation was often observed after 400 growth steps for beta cells, but only after 600 steps for alpha cells. This is exactly the opposite result than it would be obtained in case the cells were not size-normalized. Indeed, the fact that size-normalized beta cells tended to percolate sooner than alpha cells provides a clear indication that such cells tend to have more intrincated morphology. As indicated in Figure [6] which describes the cumulative two-variated distribution of dendritic segment angles, beta cells are characterized by higher dispersion of angles, implying the overall dendrites to become more disordered and spatially complex, which is in full agreement with the obtained percolation dynamics, i.e. more complex neuronal cells tend to percolate sooner than less complex cells with similar sizes. 


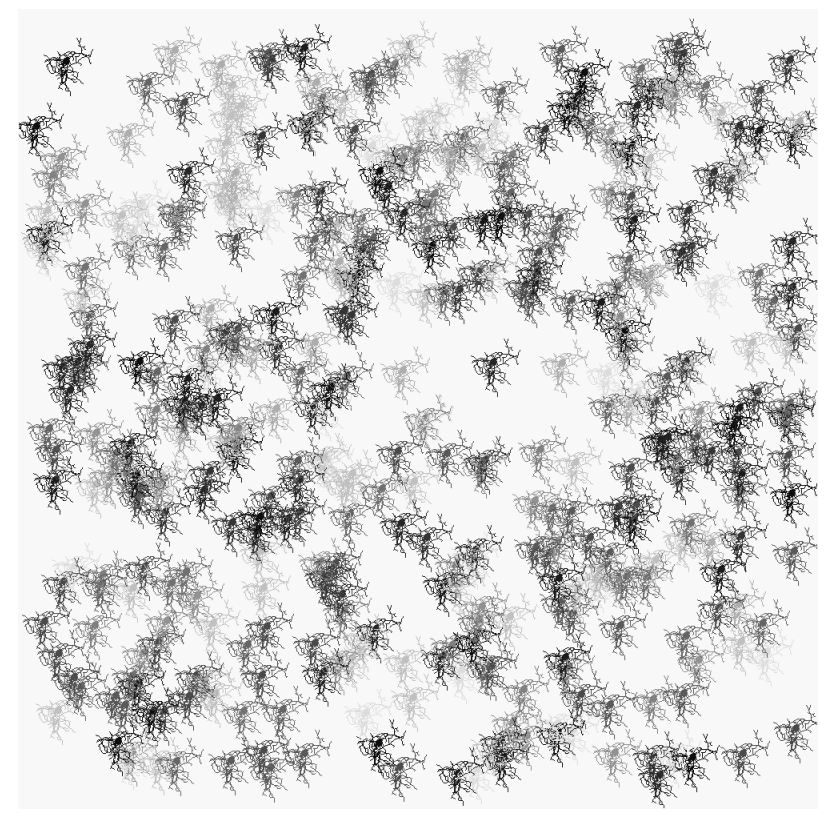

(a)

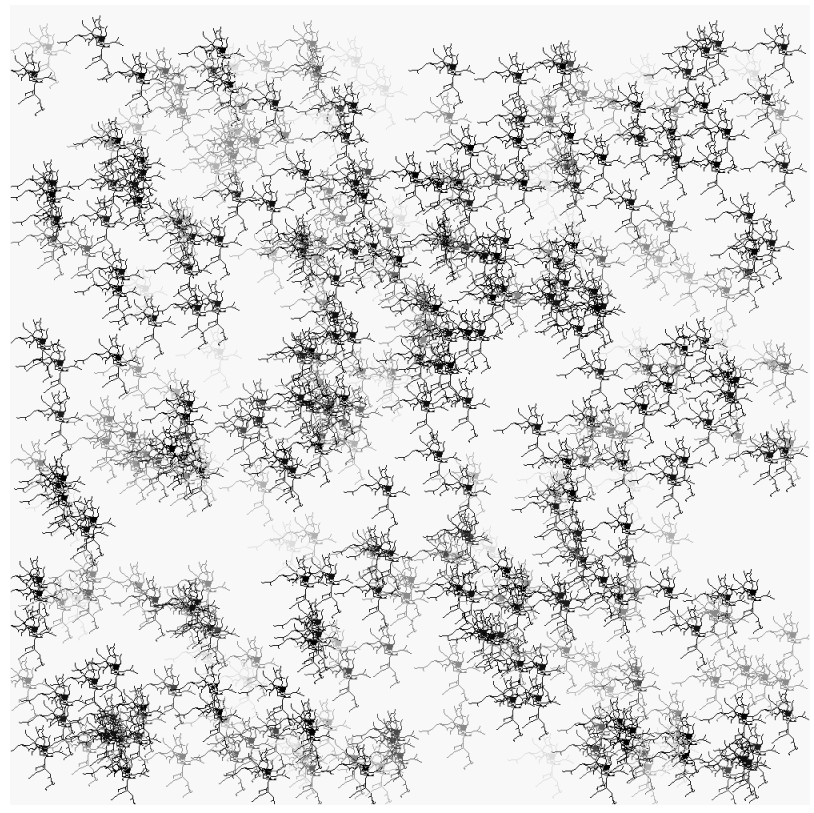

(b)

FIG. 9: Examples of neuronal networks obtained by the adopted growth methodology considering the alpha (a) and beta (b) models. Different gray levels were used for neuronal cell representation in order to facilitate the visualization of the individual cells.

\section{CONCLUDING REMARKS}

This article has reported on several new perspectives related to neuromorphic models and percolation induced by dendritic growth. First, we have shown how morphologically realistic neuronal networks can be simulated by using Monte-Carlo sampling of statistical models derived from a series of geometrical measurements of real neuronal cells. Second, we have investigated a new perspective to percolation studies in which, instead of incorporating new connections of fixed size between the involved elements, the percolation dynamics is defined by the progressive growth of dendrites/axons, following biologically-realistic rules derived from experimental data. The obtained results indicate that the percolation in such evolving systems is also characterized by abrupt transitions of the dominating cluster size along the progression of the growth and connections. We have shown that distinct critical points are usually identified for growing dynamics of systems underlain by distinct neuronal morphologies, with beta cells reaching percolation sooner than alpha cells, a result that is related to the fact that the dendritic processes of beta cells are more intrincated and spatially complex.

Such results establish and interesting connection between the statistical geometrical features of the considered cells and their potential for forming clusters among the neuronal milieu. Such perspectives and results are particularly interesting because the functional properties of neuronal networks are closely related to their connectivity (e.g. 10, 12, 18, 19]). The perspectives for further investigations are many. For instance, it would be particularly interesting to check how the consideration of more than one distinct statistical model of neuronal geometry will affect the measured critical point. Another interesting possibility is to investigate, in the spirit of [18], to what an extent the critical point statistics can be used as a resource for classification of the morphological types of involved neuronal cells. A third promising future development is to quantify, through simulations, how the geometrical properties of the neuronal cells (e.g. [19]), by controlling the sizes of the neuronal clusters, ultimately define the functional properties of the obtained structures 12]. Still, it would be interesting to correlate several measurements from complex network research, especially those related to the hierarchical structure of the networks [20], with the critical percolation time.

\section{Acknowledgments}

Luciano da F. Costa is grateful to FAPESP (processes 99/12765-2) and CNPq (process 308231/03-1) for financial support. The authors thank Luis Diambra, Marconi S. Barbosa and Gonzalo Travieso for reviewing and commenting on this work. 


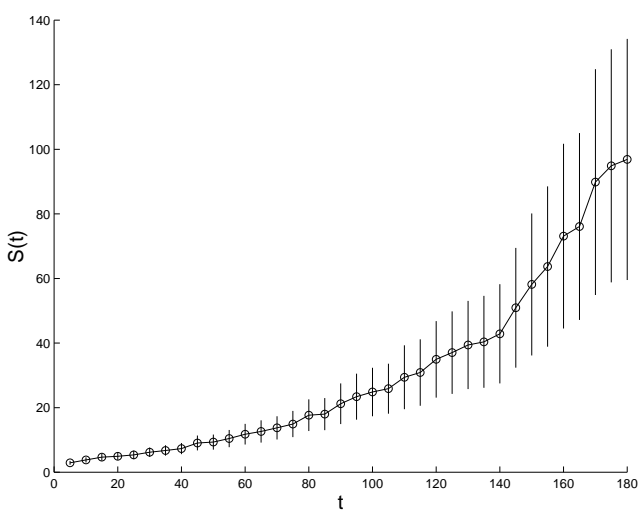

(a)

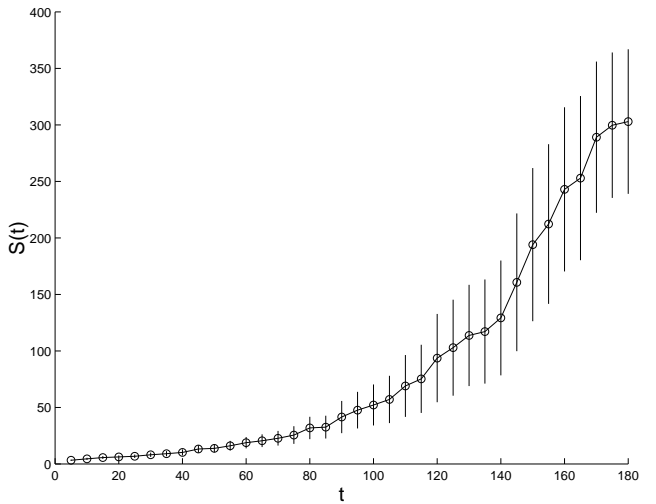

(b)

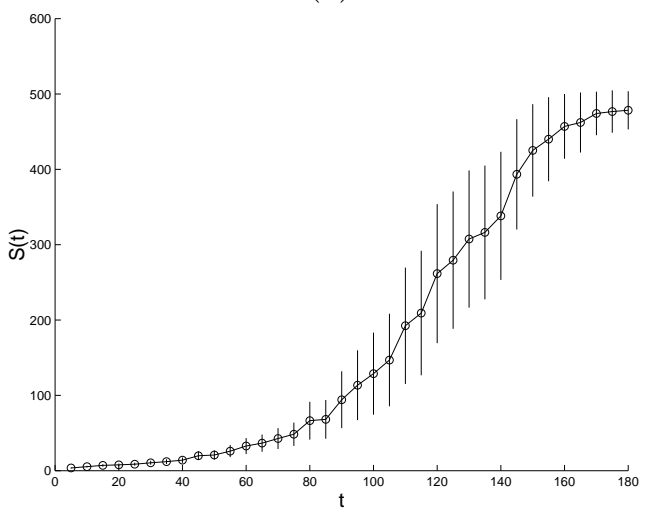

(c)

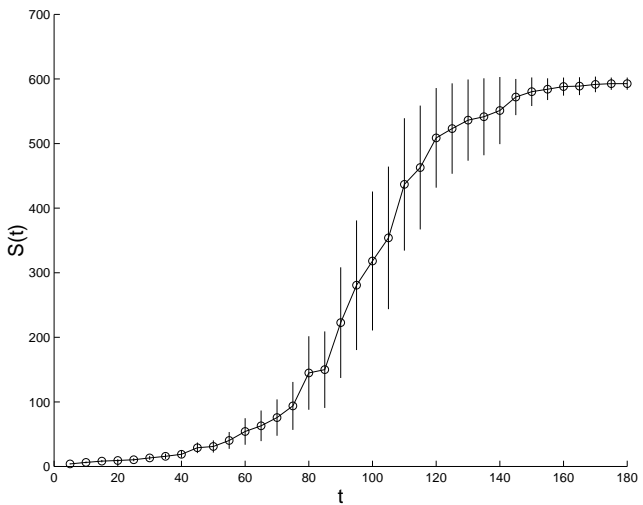

(d)

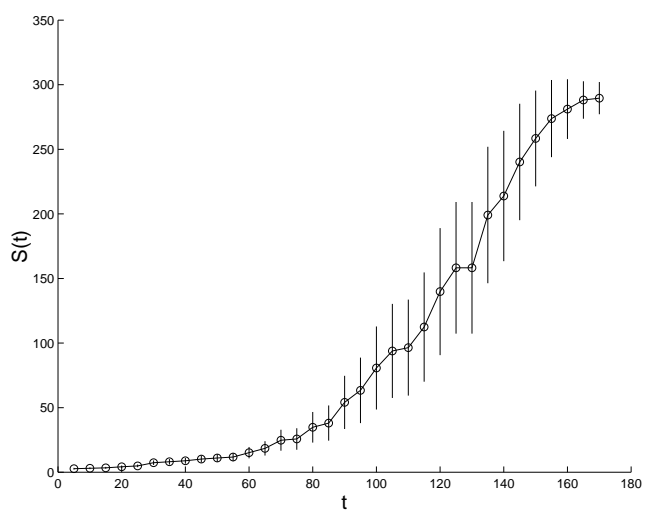

(e)

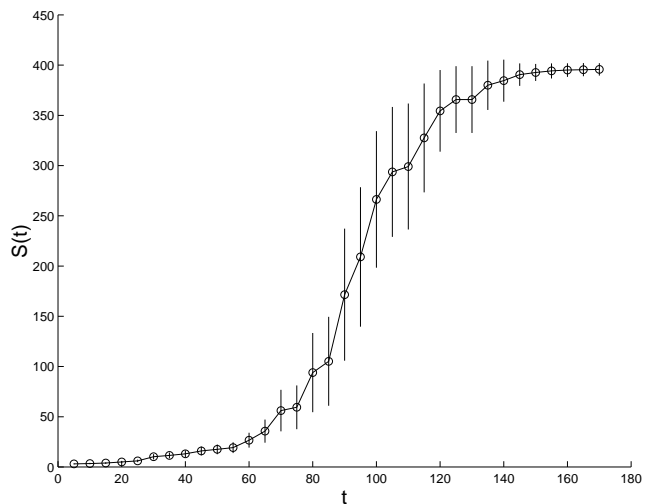

(f)

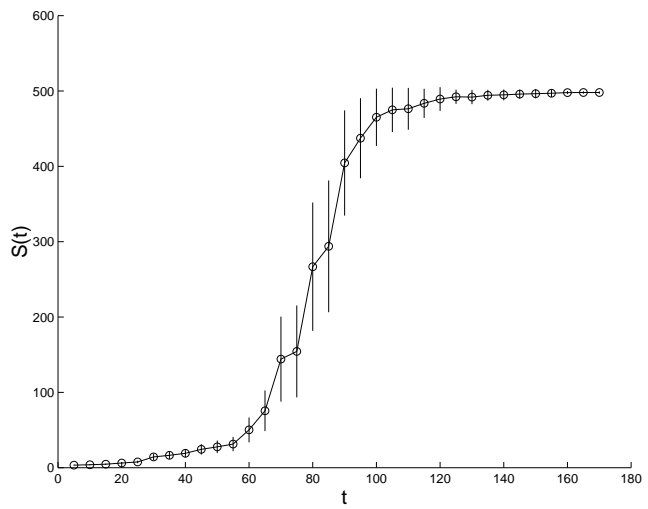

(g)

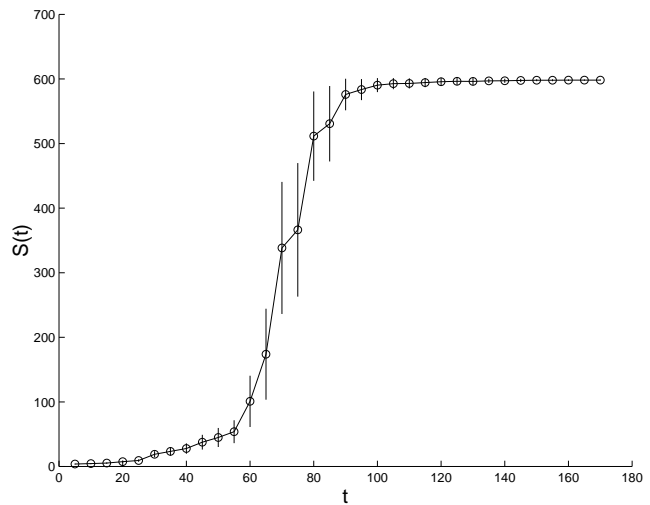

(h)

FIG. 10: Mean and standard deviation of the size of the largest cluster in terms of increasing densities (300, 400, 500 and 600 cells) of alpha (a-d) and beta (e-h) cells. 
[1] E. R. Kandel, J. H. Schwartz, and T. M. Jessel, Essentials of neural science and behavior (Appleton and Lange, Englewood Cliffs, 1995).

[2] R. Albert and A. L. Barabási, Rev. Mod. Phys. 74, 47 (2002).

[3] M. E. J. Newman, SIAM Review 45, 167 (2003), condmat/0303516.

[4] S. N. Dorogovtsev and J. F. F. Mendes, Advances in Physics 51, 1079 (2002), cond-mat/0106144.

[5] D. Stauffer and A. Aharony, An introduction to percolation theory (Taylor and Francis, 1991), second edition.

[6] J. Karbowski, Phys. Rev. Lett. 86, 3674 (2003).

[7] O. Shefi, I. Golding, R. Segev, E. Ben-Jacob, and A. Ayali, Phys. Rev. E 66, 021905 (2002).

[8] L. da F. Costa and E. T. M. Monteiro, Neuroinformatics 1, 65 (2003).

[9] R. Segev, M. Benveniste, Y. Shapira, and E. Ben-Jacob, Phys. Rev. Lett. 90, 168101 (2003).

[10] D. Stauffer, A. Aharony, L. da F. Costa, and J. Adler, Eur. J. Phys. B 32, 395 (2003).

[11] L. da F. Costa and D. Stauffer, Physica A 330 (2003).

[12] L. da F. Costa, M. S. Barbosa, V. Coupez, and D. Stauffer, Brain and Mind 4, 91 (2003).

[13] G. A. Ascoli and J. L. Krichmar, Neurocomputing 48, 1003:1011 (2000).

[14] R. C. Coelho and L. da F. Costa, Neurocomputing 48, 555 (2001).
[15] H. Waessle, H. Peichl, and B. B. Boycott, Proc. Roal Soc. London 212, 157 (1981).

[16] H. Waessle, B. B. Boycott, and R.-B. Illing, Proc. Roal Soc. London 212, 177 (1981).

[17] L. da F. Costa and R. M. C. Jr, Shape Analysis and Classification: Theory and Practice (CRC Press, Boca Raton, 2001).

[18] L. da F Costa, E. T. M. Manoel, F. Faucereau, J. Chelly, J. van Pelt, and G. Ramakers, Network: Comput. Neural Syst. 13, 283 (2002).

[19] L. da F. Costa and T. Velte, J. Comp. Neurol. 404, 33 (1999).

[20] L. da F. Costa, Phys. Rev. Lett. 93, 98702 (2004), condmat/0312646.

[21] Indeed, even the fate of neuroblasts along the cell differentiation process often does not involve genetical coding 1].

[22] This neural tracer, developed by L. A. Consularo during his $\mathrm{PhD}$ at the Cybernetic Vision Research group, allows operator-assisted tracing of the neuronal cells, producing results in the Eutectic format.

[23] Given two cells with similar morphologies but different sizes, the larger cell will obviously tend to percolate sooner.

[24] A branch point along the dendritic arborization corresponds to a point where the growing dendrite bifurctes. 


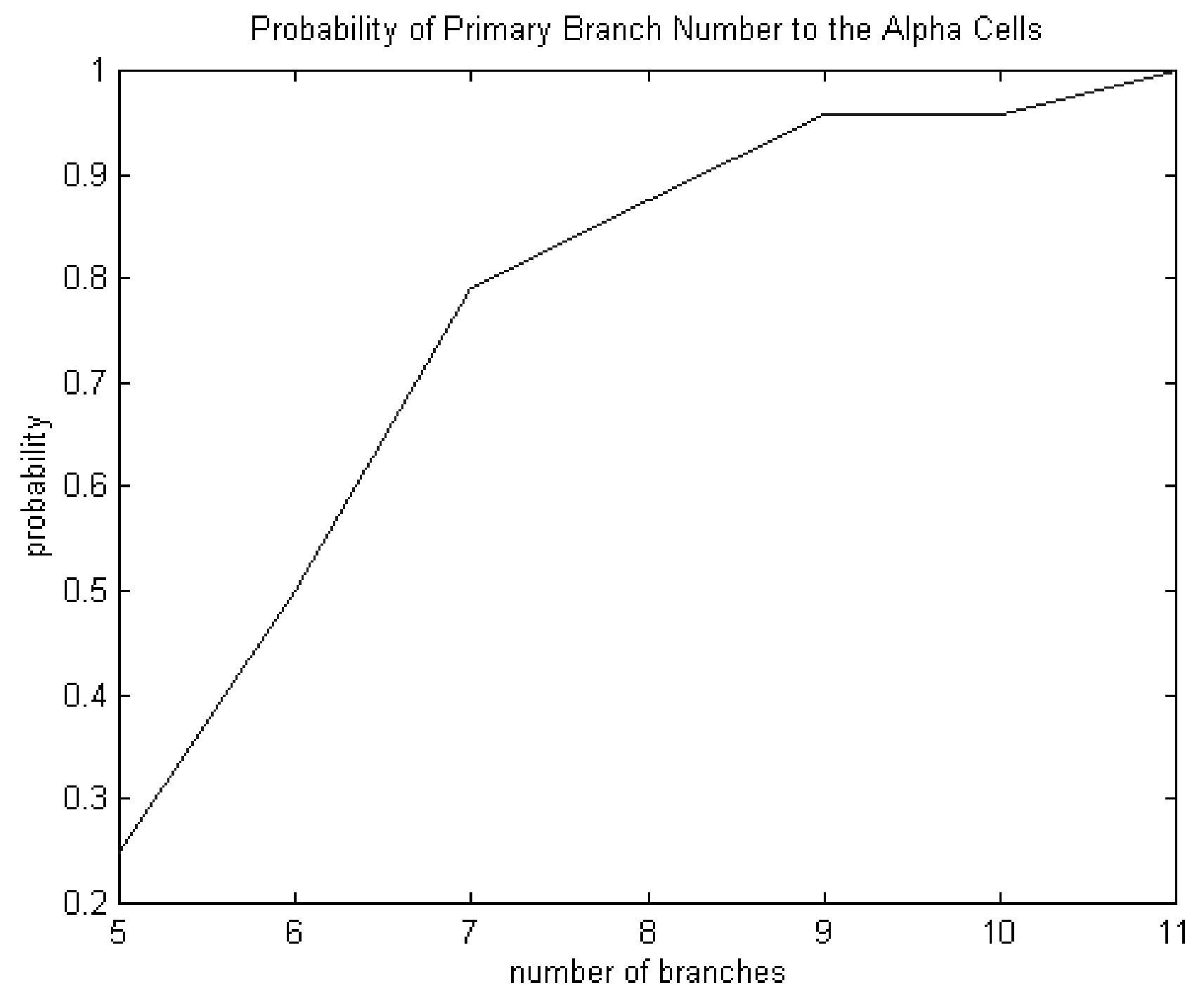




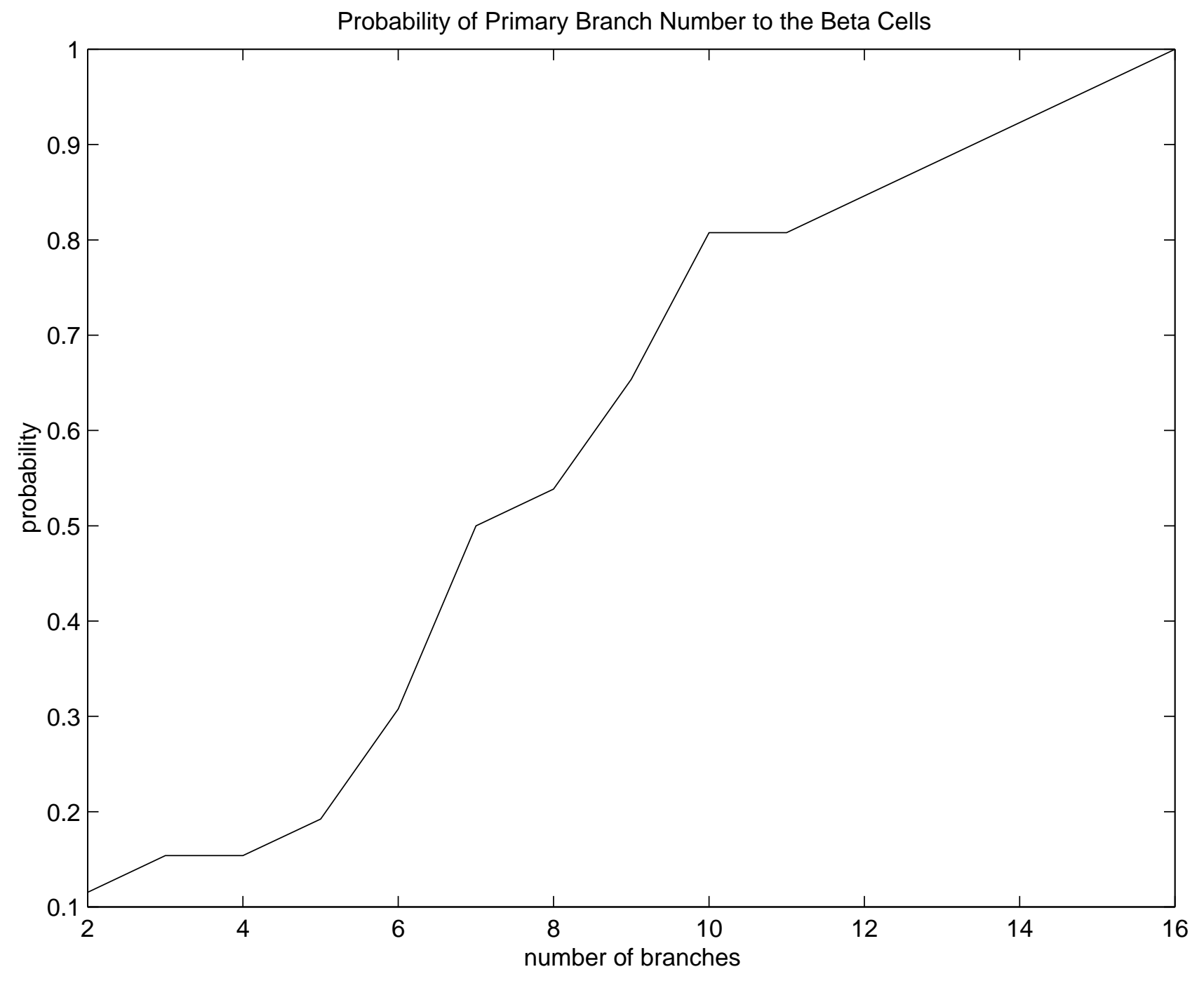

